\title{
Locativos no verbales en Mazahua (otomangue)
}

\author{
No-verbal locatives in Mazahua (Oto-Manguean)
}

\author{
Armando Mora-Bustos ${ }^{1}$
}

DOI: $10.26512 /$ rbla.v12i1.30593

Recebido em agosto/2020 e aceito em agosto /2020

\section{Resumen}

El objetivo de este trabajo se centra en la descripción y explicación de los diferentes mecanismos gramaticales que dispone la lengua para expresar la locación no verbal. La importancia de este trabajo radica en explicitar que el mazahua dispone de recursos morfológicos, léxicos y sintácticos para exhibir los sentidos locativos; esto bajo la perspectiva de que esta lengua como muchas otras lenguas otomangues es muy productiva en codificar los sentidos de locación en los predicados verbales, etiquetados como verbos disposicionales, posicionales, locativos. Las formas no verbales que expresan locación se encuentran expresadas en el nivel morfológico, el léxico y el sintáctico. Estos rasgos gramaticales pueden coocurrir dentro de uno oración; la frase locativa y la frase prepositiva son adjuntos que presentan funciones sintácticas particulares.

Palabras clave: Verbo. Frase. Preposición. Morfología. locativo.

\begin{abstract}
The aim ot this work is to describe and explain the different grammatical mechanisms that Mazahua has to express non-verbal location. The importance of this work lies in explaining that Mazahua has morphological, lexical and syntactic resources to convey location. Like meany other Oto-Manguean languages, Mazahua has a very productive way to encode location through verbal predicates, which can be dispositional, positional, locative and stative. On the other hand, there are non-verbal forms that express location at the morphological, lexical and syntactic level. There grammatical resources can co-occur within a clause and noun phrases; locative phrase and prepositional phrases are adjuncts that present specific sintactic functions.
\end{abstract}

Keywords: Verb. Phrase. Preposition. Morphology. Directional. Locative.

1 Profesor-Investigador de la Universidad Autónoma Metropolitana-Iztapalapa. Ciudad de México. E-mail: amora@xanum.uam.mx 


\section{Introducción}

El objetivo de este trabajo es describir los diferentes recursos gramaticales que dispone el mazahua para expresar locaciones; específicamente aquí se dará cuenta de las expresiones morfológicas y sintácticas que codifican locación; esto es, morfemas libres en forma de clíticos y morfemas ligados o formas lexicalizadas, frase nominal, frase prepositiva y un predicado verbal con orientación de dirección. El aporte de este trabajo está orientado en caracterizar cada uno de los recursos gramaticales que se encuentran en la lengua y mostrar las coocurrencias que se generan entre estos. La frase nominal locativa presenta una estructura compleja que se encuentra saturada por un núcleo nominal, un artículo, la marca de posesión y operadores locativos; la frase prepositiva locativa aparece modificada por diferentes tipos de complementos: uno nominal y otro verbal. El primero expresa un lugar específico, mientras que el segundo expresa un evento con dirección. El tercer recurso para expresar la locación es el verbal; un verbo direccional con un adjunto como complemento denota una subclase de expresiones locativas. De otra parte, en ciertas formas locativas lexicalizadas, la preposición locativa y verbo de dirección aparecen exclusivamente como marcas de locación y dirección respectivamente.

La variedad de mazahua objeto de estudio pertenece a San Pedro Potla, municipio de Temascalcingo; Estado de México, (ISO 639.3). Los datos pertenecen a diferentes materiales recolectados desde hace una década (historias de vida, entrevistas, narraciones, cuentos de tradición oral). Los colaboradores son mujeres y hombres, hablantes nativos de mazahua, todos ellos originarios de San Pedro Potla ${ }^{2}$.

Para entender la arquitectura de los locativos, es necesario tener en cuenta algunos rasgos relevantes de la gramática de la lengua. El mazahua presenta un sistema nominativo-acusativo y un sistema escindido en los verbos estativos y pacientivos; es una lengua de marcación en el núcleo; presenta un orden de constituyentes V O S, V S, S V O OI. La palabra verbal está integrada por marcas de quantificación, aspecto, movimiento asociado, modo, polaridad, número, énfasis, demostrativos y locativos; el mazahua presenta un repertorio de cuatro tonos: dos de nivel (alto, bajo) y dos de contorno (ascendente y descendente); por lo general, los tonos de nivel se anclan en el proclítico verbal y codifican los rasgos de tiempo, aspecto modo y persona; en el ámbito nominal codifican posesión; similar a lo que ocurre con otras lenguas otomangues, el mazahua dispone de un sistema complejo de verbos de movimiento, disposicionales,

2 Quiero agradecer por la colaboración incondicional a Josefina Ramírez y a Maurilio Ramirez de la Cruz, hablantes nativos de mazahua y originarios de San Pedro Potla. Igualmente, este trabajo se ha podido realizar gracias al apoyo constante de la esposa e hijos de Antonio López Marín. 
posicionales, locativos, etc.; esto implica que en algunas lenguas no se encuentre un repertorio de lo que en la tradición gramatical se denomina adposiciones.

Este trabajo está organizado de la siguiente manera. Clases verbales y construcciones locativas básicas; morfología locativa; formas libres o adverbios léxicos; frase nominal locativa; frase prepositiva locativa; verbo de movimiento direccional; formas léxicas; locativos y coocurrencias y conclusiones.

\section{Clases verbales y construcciones locativas básicas}

En mazahua, la expresión de la locación se realiza básicamente de forma verbal; Knapp (2008) a este respecto, propone una clasificación que consiste en verbos existenciales (posicionales) y verbos transitivos (depositivos y portativos). Esta lengua, contrario a lo que ocurre en otras lenguas de México (Bohnemeyer y Brawn, 2007), no tiene un sistema morfológico a través del cual se sistematice paradigmáticamente la locación, la posición o la disposición. Los verbos de (1) kẫrã 'haber muchos', k'ás'í 'atajar', ygós't colocar en, Pòrtì 'adelantar a', Pórì 'estar parado' expresan lexicamente la locación o la disposición; este hecho se evidencia en estas expresiones verbales al no explicitar en las formas oracionales ningún tipo de recurso morfológico o sintáctico que dé cuenta de manera alternativa de la locación, como se ven en: míkãrã nájnîni 'vivía en un pueblo' de (1a), mikk'áski n 'îhi ‘atajaba en el camino' de (1b), ßá ygós’i núhjoho 'colocó en el hombro' de (1c), rà?òrpi ní?i 'va a la cabeza' de (1d) y tá?órít 'estar parado' de (1e).
(1) a. jò=tée=k'o $\quad m i=k \hat{a} r \tilde{a} \quad n a ́=j$ nîni $\quad$ ó $=\mathrm{ts}_{\mathrm{a}}^{\mathrm{h}}=\mathrm{hi} \quad$ ná=mbá $\int w a$ ARTPL. DEF $=$ gente $=$ SUB $3 \mathrm{COP}=$ haber.reunido $\quad$ uno $=$ pueblo $3 \mathrm{PST}=$ hacer $=\mathrm{PL}$ uno $=$ fiesta 'La gente que había (vivía) en un pueblo hizo una fiesta.' (txt)

b. mí=k'ás-ki j'îhi

$3 \mathrm{COP}=$ atajar.animado-10 camino

'Me atajaba en el camino.' (txt)
c. ná=hó?o $\quad \beta a ́=\varnothing=$ ygós'i $\quad n u ́={ }^{h} j o ̌ h o$ PRED.PRS $=$ bueno $\quad \mathrm{CIS}=3 \mathrm{PST}=$ colocar.en $\quad$ ART.3POS=hombro 'Lo colocó muy bien en su hombro.'
d. já=ná=k'i rà=?òr-pi ní?i ná=k'i rà=?'̇s-pi $\mathrm{PTL}=\mathrm{uno}=\mathrm{REL} \quad 3 \mathrm{FUT}=$ adelantar.a-3DAT $\quad$ cabeza $\quad$ Uno $=\mathrm{REL} \quad 3 \mathrm{FUT}=1$ levar-3DAT $\mathrm{a}=\int \mathrm{f}^{\mathrm{h}} \mathrm{i}$ LOC $=$ espalda
'Uno que va a la cabeza y otro que lleva atrás.'

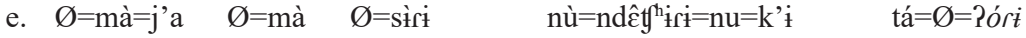 $2 \mathrm{IMP}=\mathrm{ir}=\mathrm{PTL} \quad 2 \mathrm{IMP}=\mathrm{ir} \quad 2 \mathrm{PRS}=$ atrapar $\quad \mathrm{ART}=$ borrego $=\mathrm{DEM}=\mathrm{REL} \quad$ hasta $=3 \mathrm{PRS}=$ estar.parado $n \grave{u}=k^{h} a n u$ ART.E=así:allá.PROX3
'Vete ahora, ve atrapar el borrego que está hasta allá.'


Es común encontrar construcciones como las de (2), en donde el verbo de movimiento implica una meta sin que haya una marca que la identifique como tal: járè̃e zángí indzùmì 'vino a regañarme a mi casa' de (2a), ómàa hnîni 'fue a Temascalcingo' de (2b); de otra parte, la oración zóbí ygùmì 'dejó la casa' de (2c), es intransitiva; el verbo intransitivo zóbi tiene su respectivo correlato transitivo sóyi; en esta construcción la frase nominal ngumi se manifiesta como adjunto.

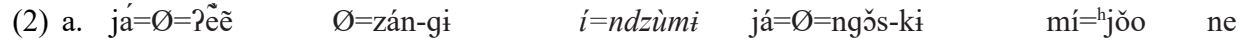 $\mathrm{PTL}=3 \mathrm{PST}=\mathrm{venir} \quad 3 \mathrm{PST}=$ regañar $-1 \mathrm{O} \quad 1 \mathrm{POS}=\mathrm{casa} \quad \mathrm{PTL}=3 \mathrm{PST}=$ acusar $-1 \mathrm{O} \quad 1 \mathrm{POS}=$ papá $\quad \mathrm{CONJ}$ mí=níi
1POS=mamá
'Vino a regañarme a mi casa, me acusó con mi papá y mi mamá.'

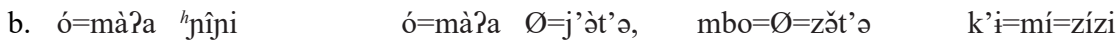
$3 \mathrm{PST}=\mathrm{ir} \quad$ Temascalcingo $\quad 3 \mathrm{PST}=\mathrm{ir} \quad 3 \mathrm{PST}=$ curar $\quad$ luego $=3 \mathrm{PST}=1$ legar $\quad \mathrm{DEM}=3 \mathrm{POS}=$ tía $\mathrm{mbo}=\varnothing=\int \hat{\mathrm{i}}-\mathrm{tsi} \ldots$
luego $=3$ PST $=$ decir -1 oI
'Fue a Temascalcingo a curar, luego llegó mi tía, luego me dijo...'

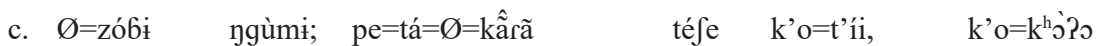 $3 \mathrm{PST}=$ dejar casa pero=hasta $=3 \mathrm{PRS}=$ vivir todo $\mathrm{DEMPL}=$ niño $\quad \mathrm{DEMPL}=$ nuera 'Dejó la casa; pero hasta viven todos los hijos, las nueras.'

Las construcciones que resultan como respuesta a la construcción locativa básica (Levinson, 2003; Levinson y Wilkins, 2006; Ameka y Levinson, 2007) son de diferente naturaleza gramatical; en (3) se ilustran ejemplos de la construcción locativa básica, preguntas-dónde; solo en (3a), se explicita la locación que da cuenta de la pregunta introducida por há 'dónde', àftith ${ }^{\text {h }} \mathrm{k}$ 'à èmiliano zàpata 'atrás en la Emiliano Zapata'; en (3b) y (3c) no se exalta la locación expresada en la respectiva construcción locativa básica directa e indirecta; discursivamente, en los dos últimos ejemplos, el cuestionamiento sobre la locación es relevante, pero la entidad locativa referida es de conocimiento común.

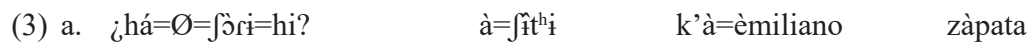
dónde $=3$ PST $=$ estudiar $=\mathrm{PL} \quad$ LOC $=$ espalda $\quad$ DEM. LOC $=$ Emiliano $\quad$ Zapata
¿¿Dónde estudiaron? Atrás, en la Emiliano Zapata.'
b. mbe $=i=$ mběne $\quad$ hhá $=\beta i=\varnothing=$ nã́ $=\beta i$ ? $\quad$ hã̃ã, $\quad$ í $=$ mběne $=\mathrm{t}^{\mathrm{h}} \mathrm{O}$
pero $=2 \mathrm{PRS}=$ recordar $\quad$ donde $=\mathrm{AMB}=2 \mathrm{PST}=$ hablar $=\mathrm{DU} \quad \mathrm{AF} \quad 1 \mathrm{PST}=$ recordar $=\mathrm{DEL}$
'Pero recuerdas ¿dónde hablaron? Sí, me acuerdo.'
c. ¿há =mí=ndzódi=nu? j’á=mí=tôt ${ }^{\prime} \mathrm{i}=\mathrm{hi}$
dónde $=3 \mathrm{COP}=$ caminar $=\mathrm{DEM} \quad \mathrm{NEG}=3 \mathrm{COP}=$ encontrar $=\mathrm{P}$
‘¿Dónde andaba ese? No lo encontraban.’

En las construcciones de (4a) y (4b) está implicada una locación deíctica, expresada igualmente en la forma há junto con el enclítico $k^{w} a$ que codifica 
locación de proximidad 2; esta forma enmarca la escena locativa en donde acontecerá un determinado hecho. La forma $h a ́ k^{w} a$ aparece básicamente en primera posición de la construcción y no expresa ningún tipo de conector clausal, ya que en los casos de subordinación locativa se usa el subordinante locativo k'a como en (4c).

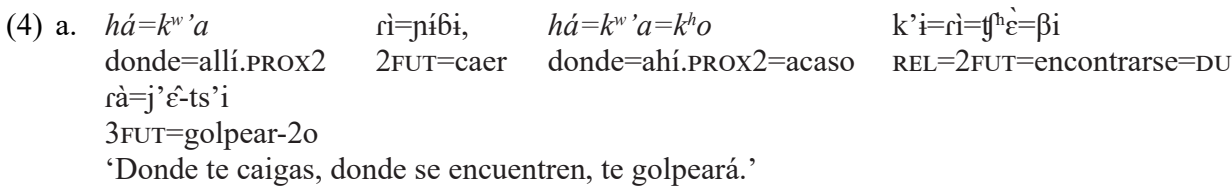
b. $h a ́=k^{w}$ 'a rì=ts ${ }^{\mathrm{h}} \hat{\mathrm{o}} 6 \mathrm{i} \quad$ ná=káro rà=ndìr-k' $\mathrm{i}$,

donde=allí.PROX2 3FUT=pasar uno=carro $\quad$ 3FUT=empujar-2o

De otra parte, en otro tipo de preguntas en construcciones locativas básicas, aparece la forma há con una marca de movimiento asociado, hè, como en (5). Este tipo de pregunta implica que la entidad expresada en el sujeto gramatical está asociada con un desplazamiento que implica un cambio de locación inherente (Guillaume 2017); dado que en este tipo de construcción locativa básica se implica por default la locación, en la respuesta se puede, como en (5a), o no, como en (5b), expresar la locación referida en la marca de movimiento asociado.

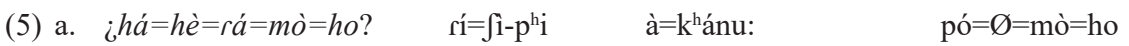 dónde $=\mathrm{LOC}=1 \mathrm{FUT}=\mathrm{ir}=\mathrm{PL} \quad 1 \mathrm{PST}=$ decir $\quad$ LOC $=$ así:allá.PROX3 $\quad$ tal vez $=1 \mathrm{FUT}=\mathrm{ir}=\mathrm{PL}$ mà $=6$ óndo ir.DIR=México
¿¿Dónde vamos a ir? Le dije así: tal vez por México.'

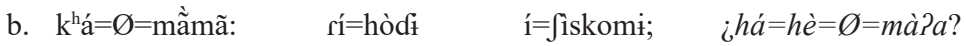 luego $=3 \mathrm{PST}=$ decir $\quad 1 \mathrm{PRS}=$ buscar $\quad 1 \mathrm{POS}=$ papel $\quad$ dónde $=\mathrm{LOC}=3 \mathrm{PST}=\mathrm{ir}$ 'Luego dijo: estoy buscando mi papel; ¿dónde se fue?'

En (6) se presentan ejemplos de las construcciones locativas básicas generadas con el predicado copulativo ygè 'ser' y la forma $h a ́ k^{w}$ ' $a$ 'dónde', ejemplificadas en (4). Estas preguntas-dónde requieren que en la respuesta esté implicada una locación expresada a través de alguno de los recursos que dispone la lengua, es decir, à $\int_{o}$ ’ník $^{w}$ ' $a$ 'allá arriba' de (6a) y mà $\int o$ 'ní àmà $k^{h} a b \partial$ 'por allá arriba, por aquí' de (6b). 


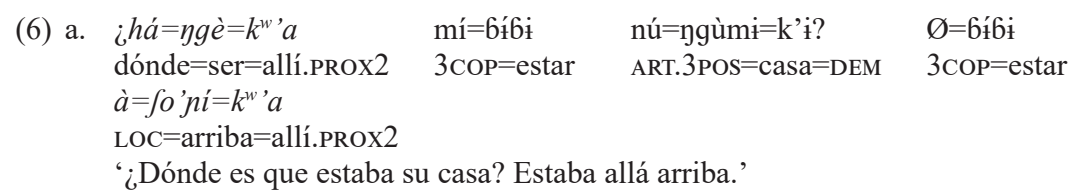

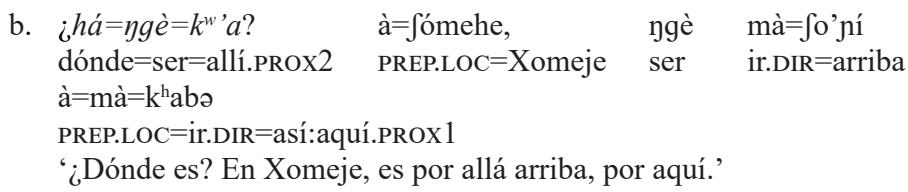

Para la descripción de las formas locativas no verbales se parte de que el mazahua pertenece a las lenguas mexicanas que presentan verbos disposicionales, posicionales, de movimiento; la pregunta de locación básica requiere de una forma $h a ́$ 'dónde', que tipifica la pregunta de locación; en las respuestas a esta pregunta está implicada una forma no verbal que expresa locación, o en otros contextos se presupone la locación; no obstante, las preguntas con la cópula predicativa, ygè, requiere que en la respuesta aparezca explicitada una forma no verbal de locación. La naturaleza semántica de los verbos está relacionada con el hecho de que la lengua presente diferentes mecanismos para configurar los sentidos de locación, tales como marcas locativas, demostrativos locativos, preposición locativa y un verbo de dirección. Estos mecanismos han sido ya descritos en otras lenguas otomangues, tales como: mazateco, amuzgo, mazahua (García, Hernández, Ortiz y Mora 2019), chichimeco (Hernández y Mora 2019), me'phaa (Hemphill y Hemphill 2019) y zapoteco de Tlacolula (Lillehaugen 2006), entre otros trabajos.

\section{Morfología locativa}

El mazahua dispone de dos juegos que codifican locación; el primero está integrado por morfemas en forma de clítico que expresan diferentes grados de distancia con respecto al punto de referencia, en el segundo se encuentran los morfemas libres o formas léxicas que igualmente expresan los tres grados de distancia; en el cuadro 1 se presentan estos dos juegos.

Cuadro 1. Formas locativas

$\begin{array}{llll} & \text { AQUÍ.PROX1 } & \text { ALLÍ.PROX2 } & \text { ALLÁ.PROX3 } \\ \text { CLÍTICOS } & \text { bə } & \mathrm{k}^{\mathrm{w}} \mathrm{a} & \mathrm{nu} \\ \text { F.LÉXICAS } & \text { nùbə } & \text { nùk }^{\mathrm{w}} \mathrm{a} & \text { nùnu }\end{array}$

En los ejemplos de (7), se ilustra la posición enclítica de los morfemas ligados bə, $k^{w} a$ y $n u$. Estas formas pueden enclitizarse directamente en la base verbal o aparecen después de la marca de número, énfasis o aspecto, esto es

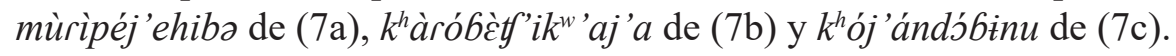


(7) a. ijo,

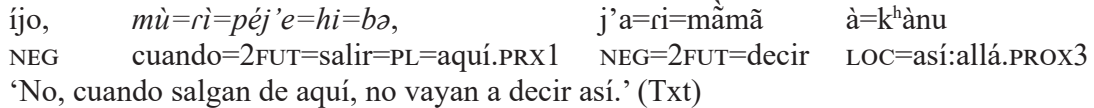

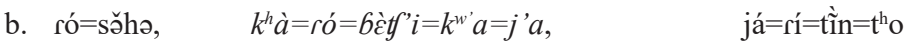
1 $\mathrm{PST}=$ llegar $\quad$ luego $=1 \mathrm{PST}=$ tomar $=$ allí.PROX $2=\mathrm{PTL} \quad \mathrm{PTL}=1 \mathrm{PST}=$ emboracharse $=\mathrm{DEL}$ 'Llegamos, luego tomamos allí, me emborraché.' (Txt)

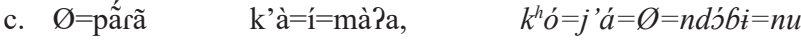
3.PRS $=$ dudar $\quad$ REL. $\cdot$ OC $=2 \mathrm{PST}=\mathrm{ir} \quad$ acaso $=\mathrm{NEG}=3 \mathrm{PST}=$ caer $=$ allá.PROX 3 'Quién sabe a dónde fuiste, acaso no se cayó allá.' (Txt)

El clítico locativo bo de (8) se enclitiza directamente en el verbo, como en rókèhmebəj'a de (8a); este clítico igualmente se puede ligar a una marca de aspecto que aparece en posición posverbal, como en ríbínt ${ }^{h}$ obə de (8b); el alcance o dominio que tiene este enclítico es sobre la unidad verbal o sobre una unidad nominal como en nùsènsioba de (8c).

(8) a. j'á=ró=bànta $\quad \mathrm{p}^{\text {hék }} \mathrm{k}^{\mathrm{h} a} \quad$ ró $=\mathrm{kèh} m e=b \partial=j^{\prime} a$

$\mathrm{NEG}=1 \mathrm{PST}=$ aguantar porqué $1 \mathrm{PST}=$ quedar=aquí.PRX $1=\mathrm{PTL}$

'No iba aguantar porque me iba a quedar aquí.' (Txt)

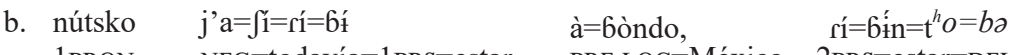

1 $\mathrm{PRON} \quad \mathrm{NEG}=$ todavía $=1 \mathrm{PRS}=$ estar $\quad$ PRE.LOC=México $\quad$ 2PRS $=$ estar $=\mathrm{DEL}=$ aquí.PRX1

'Yo todavía no estoy en México, estoy aquí.'

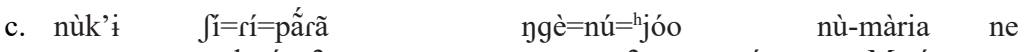

REL todavía $=3$ PST $=$ conocer $\quad$ ser $=$ ART.3POS=papá mv-María conj

$n \grave{u}$-sènsio $=$ bə

mv-Crescencio=aquí.PROx 1

'A quien todavía conocí fue a su papá de María y del Crescencio de aquí.'

En los ejemplos de (9), el morfema $k^{w^{\prime}} a$ se liga directamente al verbo, como en $k^{h}{ }^{\prime}$ rípá $^{w^{\prime}} a$ de (9a) o $\beta a ̀$ Yúnì $k^{w^{\prime}} a j$ 'a de (9c); también se adjunta a la marca de número que aparece en posición adyacente a la forma verbal, como en $o^{\prime} \mathrm{g}^{h} \dot{\varepsilon} \beta i k^{w^{\prime}} a j j^{\prime} a$ de (9b). El dominio de este locativo es verbal o nominal, como en nùsùndzek 'aj'a de (9b).
(9) a. já $=$ né $=\varnothing=j$ 'ồn-ki
$\mathrm{k}^{\mathrm{h}} \mathrm{à}=\varnothing=\int \mathrm{i}$-tsi
$k^{h} \grave{a}=r i=p \dot{a}=k^{w^{\prime}} a$
$\mathrm{PTL}=\mathrm{REC}=3 \mathrm{PST}=$ engañar $-1 \mathrm{DAT}$
luego $=3$ PST $=$ decir $-1 \mathrm{DAT}$
luego $=3 \mathrm{PRS}=\mathrm{ir}=$ allí.PRX2
'Me engaño, luego me dijo, luego voy allí.' (Txt)

b. $\quad o^{\prime}=t^{h} \dot{c}=\beta i=k^{w^{\prime}} a=j^{\prime} a$

$n \grave{u}=$ sùndse $=k^{w^{\prime}} a=j^{\prime} a$

$3 \mathrm{PST}=$ encontrar $=\mathrm{DU}=$ allí. $\mathrm{PR} \times 2=\mathrm{PTL}$

$\mathrm{ART}=$ mestiza $=$ allí.PR $2=\mathrm{PTL}$

'Se encontró allí con la señora.' (Txt)

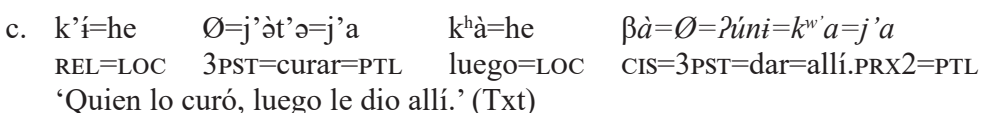


En (10), se presentan ejemplos del enclítico $n u$. De la misma manera que en los casos anteriores, este morfema se liga directamente en la unidad verbal, como en $k^{h} \grave{o j}$ 'ándj̀binu de (10b) o después de otro enclítico, como en $t s^{h} a ̆ h i n u$ de (10a). También, este clítico se adjunta a unidades gramaticales diferentes al verbo, como nùj' 'апи о 'jârinu de (10c); no obstante, el alcance o dominio está sobre el verbo.

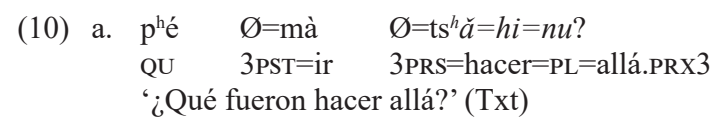

b. $\quad k^{h} \grave{o}=j^{\prime} \dot{a}=\varnothing-n d \grave{j} b \dot{i}=n u$ acaso $={ }_{\mathrm{NEG}}=3$ PST-caer $=$ allá.PR $\mathrm{X} 3$

'Acaso no se cayó allá.' (Txt)
c. nùj' $a=n u \quad$ j'á $=$ rì $=$ haandi $\quad$ hjâri $=$ nu hoy=allá.PRX3 $\quad$ NEG=2FUT=ver $\quad$ sol=allá.PRX3 'Hoy, allá no vas a ver el sol.

El sistema de clíticos locativos denota diferentes grados de distancia con respecto al centro deíctico. El espectro de visibilidad está al alcance de los interlocutores, aunque el enclítico $n u$ que codifica proximidad 3, en ciertos contextos el estado de cosas u objeto referido puede estar por fuera del alcance de visibilidad de los interlocutores del acto de habla. El alcance o dominio de estas unidades morfológicas es sobre el verbo, un constituyente de la oración o sobre toda la construcción.

\section{Formas libres o adverbios léxicos}

El sistema de clíticos, descrito más arriba, presenta un correlato de formas libres: nubə, nuk ${ }^{w} a$ y пипи. Estos adverbios léxicos codifican los mismos sentidos de distancia, con respecto al punto de referencia, que los enclíticos, esto es, proximidad 1-aquí, proximidad 2-allí y proximidad 3-allá. La función sintáctica de estas unidades gramaticales es la de adjunto (Dryer y Haspelmath 2013; Lizárraga y Mora 2015).

En los ejemplos de (11a) y en (11b) el adverbio léxico nubə aparece al final o al principio de la construcción; tiene dominio sobre la oración que implica el núcleo verbal y su o sus argumentos.
a. nùk'oró= $=\hat{i}-$ ts'i
ró=mbìta-ts'ì
mba
rá=nấ=hi
nùbə SUB 1PST=decir-2DAT 1PST=invitar-2DAT para 1FUT=hablar=PL aquí:PRX1 'Lo que te dije, te invité para que habláramos aquí.' (Txt)

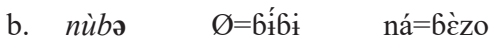 aquí:PRX1 3PRS=estar uno=señor 'Aquí está un señor.'


Estos adverbios léxicos son susceptibles a que se les adjunte un clítico de aspecto, como $n u k^{w}$ ' $a j$ ' $a$ de (12a) y (12b). Este hecho no impide que se ubiquen en posición inicial o final de oración. Tienen dominio sobre el verbo o la oración.

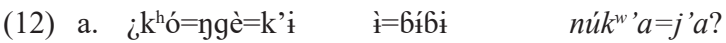
$\mathrm{QU}=\mathrm{ser}=\mathrm{SUB} \quad$ 2PRS $=$ estar allí:PRX2 $=\mathrm{PTL}$

‘¿Quién es ese con el que estás allí?’ (Txt)

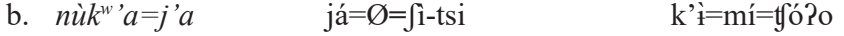
allí:PROX2 $=$ PTL $\quad$ PTL $=3$ PST $=$ decir-1DAT $\quad$ DEM $=1$ POS $=$ suegro
'Allí me decía mi suegro.'

Steward (1966) considera que 'los adverbios independientes de localidad' están formados por la partícula de enfoque $n u$ y los clíticos de localidad. Hasta el momento de esta investigación, no se ha encontrado evidencia para tener en consideración este planteamiento, por eso los adverbios léxicos antes descritos у пйп de (13a) у (13b) son analizados como adverbios léxicos. El adverbio nùnu presenta el mismo comportamiento sintáctico que nùbə y nù $k^{w}$ ' $a$; aparece al inicio o final de la oración y su dominio es sobre el predicado o la oración.

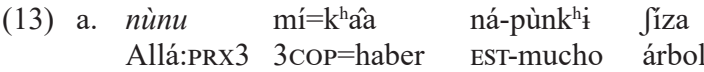

'Allá había muchos árboles.' (Elc)

b. $\quad$ ó=mà?a $\quad \varnothing=$ tũ̃ũ $\quad \grave{o}=$ zà?a $\quad$ nùnu

3PST $=$ ir $\quad 3$ PST $=$ traer $\quad$ ARTPL=leña allá:prox3

'Fue a traer leña allá.'

Los adverbios de locación deíctica están expresados en formas clíticas y en formas léxicas. Estas formas codifican tres grados de proximidad (pox1, pox 2 y pox3) con respecto al centro deíctico. Como se mostrará más adelante, los enclíticos van a coocurrir dentro de la frase prepositiva, la frase nominal locativa y con el verbo de movimiento direccional.

\section{Frase nominal locativa}

La lengua dispone de tres recursos sintácticos para expresar la locación no verbal, esto es, frases nominales locativas; frases prepositivas y un verbo de movimiento direccional que está estrechamente relacionado con las locaciones. Sintácticamente, estas unidades gramaticales, dentro de la oración, tienen el estatus de adjuntos. Los adjuntos son entendidos como todas aquellas formas gramaticales que no pueden ejercer la función de una relación gramatical (sujeto, objeto, objeto directo, objeto indirecto, objeto primario, objeto secundario); semánticamente, algunos adjuntos son susceptibles de que se les asigne un 
papel temático (Lizárraga y Mora 2015). Estas unidades periféricas (Van Valin y LaPolla 1997) pueden modificar el núcleo verbal, el verbo y argumentos o toda la cláusula.

Dentro de esta perspectiva, las frases nominales locativas de (14), $k^{\prime}$ àimp ${ }^{h}$ ême de (14a), k'ànù $k^{h}$ ons $^{\prime} i$ de (14b) y k'àj' ínze de (14c) aparecen en construcciones con verbos intransitivos. El verbo póo de (14a) es un verbo intransitivo que denota 'tener líquido'; por su parte, en (14b) y (14c) aparecen las formas intransitivas de los verbos $k^{h} \hat{o} b \dot{t}$ 'pasar' y $d a \hat{k} k \dot{t}$ 'brincar'. Estos verbos tienen su respectivo correlato transitivo: $k^{h} \hat{o g} i$ y dâsi respectivamente.
(14) a. $\mathrm{k}^{\mathrm{h}} \mathrm{ó}=\int \mathrm{i}=\mathrm{ja}$
ygè $=\mathrm{t}^{\mathrm{h}} \mathrm{O}$ ndéhe $\mathrm{k}$ 'ò $=\varnothing=$ póo
$k^{\prime} \grave{a}=i n=p^{h} \hat{e m e}$
como=todavía $=\mathrm{PTL} \quad$ ser $=\mathrm{DEL}$ agua $\quad \mathrm{REL}=3 \mathrm{PRS}=$ tener.líquido $\quad$ DEM.LOC=1POS=estómago 'Como si ya fuera agua lo que tiene mi estómago.'

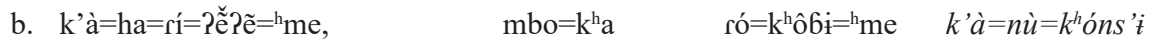 donde $=\mathrm{LOC}=1 \mathrm{COP}=$ venir $=\mathrm{PL} . \mathrm{EXCL} \quad$ luego $=$ después $1 \mathrm{PST}=$ pasarPL $\quad$ DET.LOC $=\mathrm{ART}=$ cerca 'Por donde veníamos, luego pasamos la cerca.'

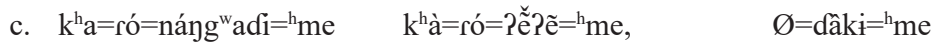 as $1=1 \mathrm{PST}=$ correr $=\mathrm{PL} . \mathrm{EXCL} \quad$ asi $=1 \mathrm{PST}=\mathrm{venir}=\mathrm{PL} . \mathrm{EXCL} \quad 3 \mathrm{PST}=$ brincar $=\mathrm{PL} . \mathrm{EXCL}$ k'à=j'înze DEM.LOC $=$ zanja
'De esta manera, corrimos y nos venimos, brincamos en la zanja.'

Las frases nominales locativas, también, aparecen en construcciones transitivas de (15a), ditransitivas de (15b) y ambitransitivas o lábiles de (15c). En todos estos contextos la posición de este tipo de frase es la posverbal. Aquí es importante resaltar que, en las construcciones ditransitivas, como en (15b), la frase que hace referencia cruzada con el sufijo de dativo $p^{h} i$ expresa una locación y en consecuencia está expresada en una frase nominal locativa, $k$ 'anuygumi 'la casa', esto muestra que el objeto indirecto también está expresado en una frase locativa (Mora y Mora 2018). En (15c), el verbo pàf 'barrer' aparece en una construcción intransitiva, la frase nominal locativa $k$ 'àtȟii nùngùmí aparecen dos entidades que establecen una relación no eventiva de locación-tema.
(15)

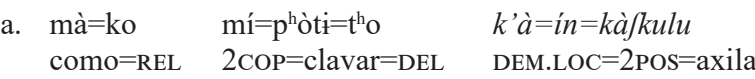 $\mathrm{i}=\int$ ískomi
2POS=papel
'Como lo clavaste en tu axila y llevaste tu papel.'

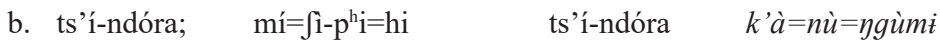 DIM-durazno $3 \mathrm{COP}=$ decir-3DAT $=\mathrm{PL} \quad$ DIM-durazno $\quad$ DEM. $. \mathrm{LOC}=\mathrm{ART} \cdot \mathrm{RCB}=\mathrm{casa}$ 'Duraznito; le decían duraznito a la casa.'
c. nù-kìna $\quad \varnothing=$ pàfí $\quad k^{\prime} \grave{a}=t^{h} \grave{\imath} i$ MV-Macrina 3 PRS $=$ barrer $\quad$ DEM. $. \mathrm{LOC}=$ afuera $\quad$ ART.RCB $=$ casa 'Macrina barre afuera de la casa.'


Algunos de los papeles semánticos que se configuran en las construcciones con frase nominal locativa son: meta, como en (16a), origen, como en(16b) y locación, como en (16c). No se debe perder de vista que los roles temáticos dependen crucialmente del estado de cosas en donde una determinada entidad está involucrada; sintácticamente, los roles semánticos de (16) son adjuntos.
a. rá=mà?a $\quad \varnothing=$ kằn-k'
1FUT=ir 1 FUT $=$ echar-2DAT
$k^{\prime} \grave{a}=n \grave{u}=\beta a ̀ m k o$
DET.LOC $=$ ART.RCB $=$ banco
'Iré a echártelo al banco en Atlacomulco.' (Txt)
b. $\mathrm{k}^{\mathrm{h}} \mathrm{à}=$ =ó=?ễhẽ, ró=péj'e $\quad k^{\prime} \grave{a}=n d z u ̀ m i=j ' a$
apenas $=1$ PST $=$ venir $\quad 1$ PST $=$ salir $\quad$ DEM.LOC $=1$ POS.casa $=$ PTL
'Apenas vine, salí de mi casa.' (Txt)
c. rì=mími $\quad k^{\prime} \grave{a}=\grave{i n}=n d z \grave{u} m \dot{t}$
2FUT $=$ sentarse DEM.LOC $=2 \mathrm{POS}=$ casa
'Te vas a sentar en tu casa.' (Txt)

En (17) se ejemplifica la estructura de la frase nominal locativa. Todas estas frases nominales locativas están formadas por un nominal, un morfema de referencia o posesión que se proclítiza y una marca de énfasis o aspecto que se enclitiza; igualmente aparece una unidad léxica que expresa locación; el demostrativo locativo $k^{\prime} a$ se ubica en posición inicial de frase, como en k'àmbóro ińndzùmíro y k'àfês'e ts 'íméhe de (17a), k'à ng áála khóns'ijj'a de (17b), k'àndìr bì ygùmì nùlùlu de (17c) y k'ànd́dehe nùndàre de (17d). El templete de la estructura de la frase nominal locativa será descrito más adelante.
(17) a. mí-hàrə
$3 \mathrm{COP}=$ construir
k'à=mbó?o
DEM.LOC $=$ dentro
ín=ndzùmi $=$ yo à $=$ ndáro,
$1 \mathrm{POS}=\mathrm{casa}=1 \mathrm{E} \quad$ LOC $=$ piedra

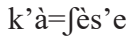
ts'î-méhe
DIM-pozo
'Estaba construida debajo de mi casa la piedra, arriba del pocito.'

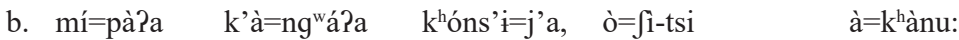
$3 \mathrm{COP}=\mathrm{ir} \quad$ DEM.LOC $=$ pie $\quad$ cerca $=$ PTL $\quad 3 \mathrm{PST}=$ decir-1DAT $\quad$ LOC $=$ así
rò $=\mathrm{mà}=\mathrm{k}^{\mathrm{h}} \mathrm{o} \quad \emptyset=\mathrm{dŭ}^{\mathrm{h}} \mathrm{mi}$
$1 \mathrm{PST}=\mathrm{ir}=1 \mathrm{E} \quad 1 \mathrm{PST}=\mathrm{sembrar}$
'Iba al pie de la cerca, me dijo así: me fui a sembrar.'
c. $\varnothing=6 \dot{6} 6 \dot{a}$ k’à=ndí?6i ygùmi nù-lùlu
3PRS $=$ estar DEM.LOC $=$ abajo casa MV-Lourdes
'Vive abajo de la casa de Lourdes.'
d. Ø=hô?ə ${ }^{\mathrm{h}}$ mố?õ $\quad \mathrm{k}$ 'á=ndéhe nù=ndàre 3PRS=haber pescado DEM.LOC $=$ agua $A R T . R C B=$ río 'Hay pescado en el agua del río.'

De la misma manera como suele ocurrir con los artículos y demostrativos (Mora y Mora 2018), un tono alto se configura sobre el demostrativo locativo, 
como en (18), para codificar posesión; la relación de posesión que se establece entre la entidad poseída y el poseedor se exhibe en el demostrativo $k^{\prime} a ̀$, como en k'àygùmí nij 'óbe adela 'en la casa de mi hermana Adela' de (18a) y en k'àygùmi nùtòna 'la casa e Antonia' de (18b); el locativo $k$ 'à aparece con tono alto.
3PST $=$ venir $=$ LIM
mí=hềnk'i;
TADV=hace.rato

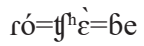
ní=j'óbe àdèla
1 Pos=hermano Adela
'Vino hace rato; la encontré en la casa de mi hermana Adela.'

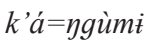
b. $\mathrm{já}=\varnothing=$ mằmi
PTL $=3$ PST $=$ decir
nu-tona,
MV-Antonia
k'ì=í=màle=j'a:
já $=\varnothing=$ mà?a
$\mathrm{DEM}=1 \mathrm{POS}=\mathrm{abuela}=\mathrm{PTL}$
a= =o'ní
LOC=arriba
'Dijo mi abuelita: se fue a la casa de Antonia, arriba.'

Estructuralmente, la frase nominal locativa está formada por el núcleo nominal, un proclítico de posesión o un artículo de referencia identificable, una forma léxica que expresa locación y el demostrativo locativo; el templete de esta frase está ilustrado en (19)

\section{DEM.LOC $(\mathrm{POS})=\mathrm{LOC} \cdot \mathrm{LEX}=\mathrm{ART} \cdot \mathrm{RCB} / \mathrm{POS}=\mathrm{NOM}=\mathrm{E} / \mathrm{ASP}$}

De (20) a (25) se han retomado algunos ejemplos de frases nominales locativas que corresponden a las construcciones (14) a (18). Las frases de (20) son simples, están formadas por un nominal nuclear y el demostrativo locativo, (20a); el nominal, más un artículo identificable y el demostrativo en (20b), y el nominal, más el enclítico de posesión y el demostrativo, en (20c).

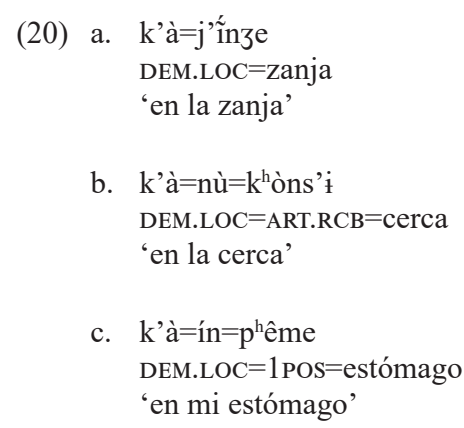

Las frases nominales locativas de (21) son complejas; están formadas por el núcleo nominal, un locativo léxico (ngwá?a 'pie' de (21a), mbóro 'adentro' de (21b), $n d^{\prime} t^{2} b \dot{ } \dot{t}^{\prime}$ 'abajo' de (21c)) y el demostrativo locativo $k$ ' $a$ que se ubica en primera posición de frase. 
(21) a. k'à=ng'á?a k k'òns'i=j'a

DEM.LOC $=$ píe $\quad$ cerca $=$ PTL

'en el píe de la cerca
b. k'à=mbó?o ín=ndzùmi $=$ yo
DEM. $. \mathrm{LOC}=$ dentro $\quad 1 \mathrm{POS}=\mathrm{casa}=1 \mathrm{E}$
'debajo de mi casa'
c. k'à=ndí?6i ngùmi nù-lùlu
DEM.LOC=abajo casa MV-Lourdes
'abajo de la casa de Lourdes'

El locativo léxico que está adyacente al demostrativo locativo $k$ 'a no puede aparecer como núcleo de una frase nominal locativa, como en (21a'), (21b') y (21c'). Dentro de este grupo aparecen nominales, algunas partes del cuerpo y adverbios.
(21) a'. *k'à=ng'wá?a
b'. *k'à=mbó?o
c'. *k'à=ndí?6ì

En (22) se ejemplifica la relación semántica que se establece entre dos entidades; la entidad poseída se encuentra con el demostrativo locativo y la entidad poseedora está en una relación de yuxtaposición. En los contextos en donde se requiera explicitar al poseedor, este se codifica a través de un tono alto que se adjunta en el demostrativo locativo.
(22)
a. k'à=ygùmi ni=j'o6e àdèla
DEM.LOC.3POS=casa 1pos=hermano Adela
'En la casa de mi hermana Adela'
b. k'à=ygùmi nù-tòna
DEM.LOC.3POS=casa mv-Antonia
'En la casa de Antonia'

En la tabla 2 se muestra el sistema de demostrativos de la lengua. Esos están clasificados a partir del número y del grado de proximidad que codifican con respecto al centro deíctico (Mora y Mora 2018). El demostrativo locativo $k^{\prime} a$ no codifica número ni distancia.

Tabla2. Juegos de demonstrativos

$\begin{array}{lll}\text { DEM } & \text { SG } & \text { PL } \\ \text { PRX } & =\text { nù } & =\text { jò } \\ & \text { nu= } & \\ & \text { nù̀nù } & \text { nùhjò } \\ \text { PRX2 } & =\text { k'i } & =\text { kò } \\ & \text { k'ì= } & \text { kò= } \\ & \text { nùk' } & \text { nùkò } \\ \text { Loc } & \text { k'a } & \end{array}$


Hasta ahora, con respecto a la frase nominal locativa, se puede mencionar que sintácticamente se realiza como un adjunto y como tal puede modificar el núcleo de la oración o toda la construcción; semánticamente, se le asignan diferentes papeles temáticos. Estructuralmente, el núcleo de una frase locativa es un nominal que coaparece con formas que codifican referencia, posesión, locación léxica y locación; el demostrativo locativo aparece en primera posición de frase.

\section{Frase prepositiva}

Aquí se entiende como adposición a la categoría gramatical que sintácticamente se constituye como la unidad nuclear-predicativa de una frase; sus complementos pueden ser de diferente naturaleza, esto es, nominal, verbal, adverbial entre otras unidades; a nivel oracional estás unidades se realizan como adjuntos subcategorizados o no por el verbo (Guerrero 2019; Hagège 2010; Van Valin y LaPolla 1997). En mazahua, el proclítico $a$, como en (23), sintácticamente funciona como preposición. Esta preposición generalmente da cuenta de lugares específicos y conocidos por los interlocutores del acto de habla. La preposición $a$ aparece con topónimos como en àbòndo 'en México' de (23a) y àmăi $/ i$ 'Manzana Larga' de (23b); igualmente, la preposición $a$ aparece con complementos que denotan lugares comunes o familiares y altamente referenciados por los individuos que participan en el evento comunicativo, como en (23c), àh̀̀mì 'en la tierra'.

(23) a.
ygè $=\mathrm{k}$ 'o $\quad$ rí $=\mathrm{k}^{\mathrm{h}} \mathrm{a}={ }^{\mathrm{h}} \mathrm{me}$,

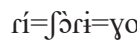
$\grave{a}=6 \grave{n}$ do
ser $=$ REL $\quad 1$ PRS $=$ hacer $=$ PL.EXCL 1 PRS $=$ estudiar $=1 \mathrm{E}$
PREP.LOC $=$ México
'Es lo que hacemos, estudio en México.'
b. nè $\quad \mathrm{k}$ 'ì=nù=pàle $\quad \grave{a}=m a \check{a}-i f i, \quad \quad$ ómbắrã $=\mathrm{k}$ 'o CONJ DEM $=$ ART.RCB $=$ abuelo $\quad$ PREP.LOC $=$ largo-manzana $\quad 3$ PST $=$ conocer $=$ DEM 'Y al abuelo de Manzana Larga, lo conoció.'
$\begin{array}{ll}\text { c. } \quad \text { ¿ó=zắn-k'í } & \text { hẵã } \\ \text { 3PST=regañar-2DAT } & \mathrm{AF} \\ \text { hó=mí=tî̀? } & \\ \mathrm{ASE}=3 \mathrm{COP}=\text { emborracharse }\end{array}$
¿¿Te regañó? Sí, me regañaba, rascaba en la tierra; estaba borracha.'

Al contenido semántico de estas frases prepositivas se les puede asignar diferentes roles temáticos, es decir meta, como (24a), trayecto o ruta, como en (24b) y locación, como en (24c).

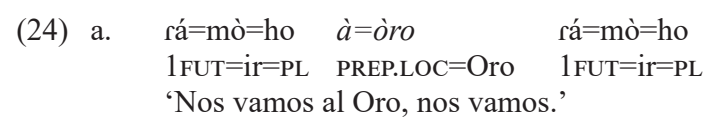




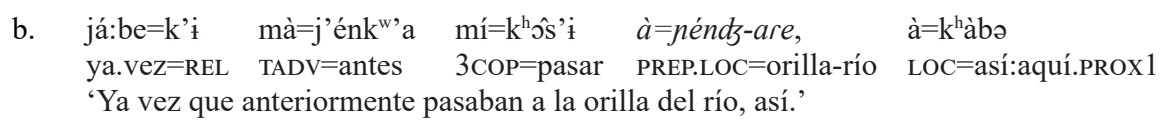

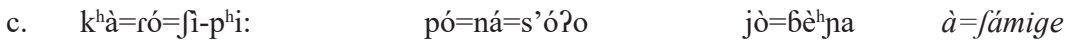
luego $=1$ PST $=$ decir -3 DAT $\quad$ tal veZ $=$ PRED.PRS $=$ malo $\quad$ ARTPL $=$ señora $\quad$ PREP.LOC $=$ Acambay à=mànu LOC=DIR:allá.PROX3
'Luego le dije: tal vez son malas las señoras de Acambay, de por allá.'

Estructuralmente estas frases prepositivas locativas, están formadas por la preposición predicativa $a$ y como argumento de la preposición aparece un nominal que denota un lugar cuya identidad es reconocida o identificada por los interlocutores del acto de habla; más adelante se describirá que esta preposición también tiene como complemento a un predicado verbal.

En (25) y (26) se presentan ejemplos para contrastar los contextos en donde aparecen una frase nominal locativa y una frase prepositiva locativa. En $k$ 'àts 'î̉já 'en la peñita' de (25a) y àt'èhe 'en el monte' de (25b) aparecen como adjuntos, en cada oración, del verbo óts'às' $i$ 'atajar'. En el primer ejemplo se está dando cuenta de un lugar indeterminado en el que ocurrió el evento (locación general), mientras que, en el segundo caso, se da como un hecho que el lugar es identificado y conocido por los interlocutores del acto de habla (locación referencial específica). No obstante, en los dos ejemplos de (25) se trata de dos construcciones sintácticamente diferentes.

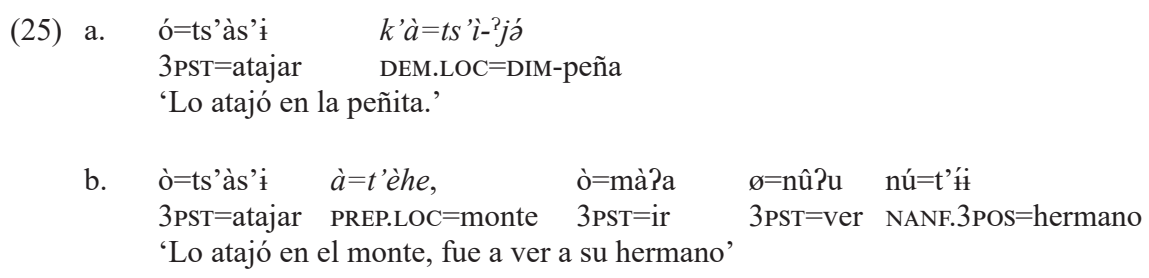

Si bien en (26) la traducción libre es la misma, 'debajo de mi casa' tanto para k'àmbó?o ínndzùmíyo de (26a), como para (26b) àmbóro ìnndzùmíyo, la estructura de estas frases es diferente. En el primer caso se trata de una frase nominal locativa compleja que está formada por un núcleo nominal, una marca de posesión, una marca de locación léxica y el demostrativo locativo; mientras que en el segundo caso, se trata de dos constituyentes que se encuentran en una relación de yuxtaposición: àmbópo y ínndzùmízo; es importante señalar que àmbópo es una forma léxica que como se mostrará más adelante tiene autonomía léxica, es decir, puede aparecer de manera independiente indicando locación; recuérdese que no ocurre lo mismo con *k'àmbóro, ya que no aparece de manera aislada en función de adjunto. 


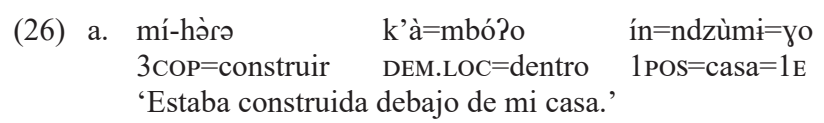

$\begin{array}{lll}\text { b. mí-hàrə } & \text { à=mbó } & \text { ín }=\mathrm{ndzùmi}=\mathrm{yo} \\ 3 \mathrm{COP}=\text { construir } & \text { LOC=dentro } & 1 \mathrm{POS}=\mathrm{casa}=1 \mathrm{E} \\ \text { 'Estaba construida debajo de mi casa.' } & \end{array}$

En los ejemplos de (27), la preposición $a$ aparece en formas léxicas que denotan locación. Estas formas se caracterizan porque están formadas por $a$ más una palabra que no tiene autonomía sintáctica, es decir, no puede aparecer en una oración como una unidad léxica independiente. Estas formas adquieren sentido sólo cuando aparece proclitizado el morfema $a$ o $m a$ (esto será descrito más adelante), esto es, los locativos $\int \hat{t}^{h} \dot{t} \dot{t}$ de (27a), nd't $26 \dot{t} d e$ (27b), th $t^{h} i$ de (27c) y fès'e de (27d), entre otros, aparecen en el repertorio léxico de la lengua junto a la preposición $a$. $\mathrm{ASE}=\mathrm{NEG}=3 \mathrm{COP}=$ dejar $-10 \quad 3 \mathrm{PST}=$ seguir $\quad$ LOC $=$ espalda $\quad \mathrm{NEG}=$ todavía $=3 \mathrm{COP}=$ dejar $-1^{\circ}$ 'No me dejaba, seguía atrás, todavía no me dejaba.'

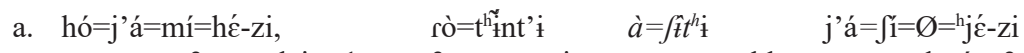

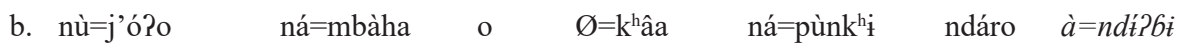 ART.DEF=perro PRED.PRS $=$ rojo $\quad$ CONJ 3PRS $=$ haber $\quad$ PRED $=$ mucho piedra LOC $=$ abajo 'El perro es rojo o hay muchas piedras abajo.'
c. nù=j'ó?o ó=mbéj'e $\quad \grave{a}=t^{h}$ hri ART.RCB $=$ perro $\quad 3$ PST $=$ salir $\quad$ LOC $=$ fuera
'El perro salió afuera.'
d. o mù= rà=tốhõ
$\mathrm{CONJ}=$ cuando $=3 \mathrm{FUT}=$ cantar $\quad$ ART.DEF $=$ DIM-pájaro
'O cuando canta el pajarito que se para arriba.'

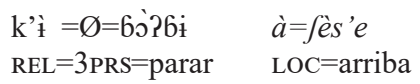

La frase nominal locativa y la frase prepositiva locativa son dos construcciones que sintácticamente funcionan como adjuntos, semánticamente pueden expresar un sentido aparentemente similar, pero difieren en su estructura. La preposición pierde su estatus nuclear y codifica locación cuando aparece en formas léxicas que denotan locación.

\section{Verbo de movimiento}

La unidad léxica mà?a corresponde a la categoría gramatical de verbo de movimiento, como en (28a); es un verbo intransitivo que por lo general requiere de un complemento que aparece en función de adjunto. Dentro de este trabajo, se ha considerado que este predicado es una pieza gramatical que hace parte del sistema de locación de la lengua; la relación que presenta con la preposición à y con las frases nominales locativas es muy significativa. En (28a) el predicado verbal intransitivo màra denota sentido de verbo de movimiento; como 
todos los verbos en mazahua aparece en su forma de cita, esto implica que está flexionado. En las construcciones mày'éndzarèkw'aj'a 'por la orilla del río, por allí', de (28b); màhnîni 'por Temascalcingo' de (28c); y màn 'ít'ehe 'por la punta del monte', de (28d), el verbo mà?a se ha neutralizado en tercera persona presente, de ahí que no aparezca el respectivo proclítico que codifica estos rasgos gramaticales. Igualmente, en estos contextos, este verbo denota un sentido de movimiento con dirección y aparece con un adjunto que denota lugar.
a. nù=ts'ít'i
$\grave{o}=m a \grave{r} a$
ART.RCB $=$ DIM-niño 3 PST $=$ ir
ná $=$ hế $=\mathrm{j} ' \mathrm{a}=\mathrm{nu}$
PRED $=$ lejos $=$ PTL $=$ DEM.PROX 1
'El niñito se fue lejos.'

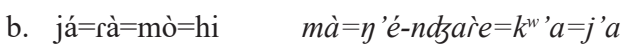

$\mathrm{PTL}=3 \mathrm{FUT}=\mathrm{ir}=\mathrm{PL} \quad$ ir.DIR $=$ orilla-río $=$ allí.PROX $2=\mathrm{PTL}$

'Ya se van a ir por la orilla del río, por allí.'

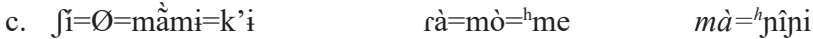
todavía $=3$ PST $=$ decir $=$ DEM $\quad 3$ FUT $=\mathrm{ir}=$ PL.EXCL $\quad$ ir.DIR $=$ Temascalcingo
'Todavía dijo ese vamos por Temascalcingo
d. è-fùba ò=j'ês'i jò=ndtîni $\quad m \grave{a}=n$ 'í-ty'èhe
MV-Juan 3PST=arrear ARTPL.RCB=toro ir.DIR=cabeza-monte
'Juan llevó los toros por la punta del monte'

De la misma manera de lo que ocurre con la preposición à, la forma mà?a también aparece en formas léxicas, como en (29), màjât $t^{h} \dot{\mathbf{i}}$ de (29a), màhòmì de (29b), màn 'áng ${ }^{w}$ arì de (29c), màndí? $6 \dot{t}$ de (29d). En todos estos casos ma?a codifica dirección. Las formas que se adjuntan $\int \mathrm{fth}^{h} \dot{\mathrm{i}}$, hòmi, n’ángwari y ndí? $6 \dot{t}$ denotan el sentido de locativo; recuérdese que estas formas léxicas no pueden aparecer de manera independiente en los contextos en donde se genera este sentido.
a. já $=$ rì $=$ đầs' $\mathrm{i}=\mathrm{t}^{\mathrm{h}} \mathrm{O}$,
mbe $=$ tí $=\varnothing=$ mbít'i
$\varnothing=$ ndzôbi
$m \grave{a}=\int \hat{t} t h \grave{i}$
PTL $=3$ PST $=$ brincar $=$ DEL
pero $=\mathrm{MOV}=3$ pst $=$ temblar
3PST=regresar
DIR=atrás
'Ya brinco, pero regreso temblando por atrás.'

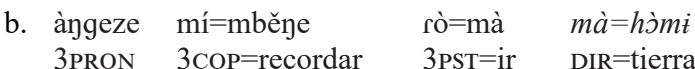

'Él recordaba que iba por abajo.'

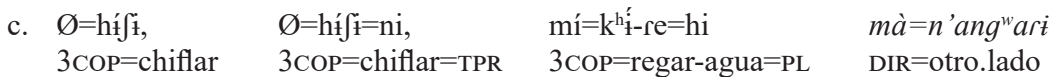
'Chiflaba, chiflaba, regaban por el otro lado.'

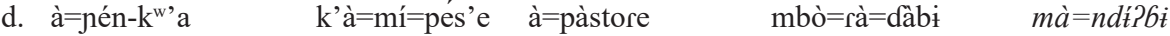 LOC=lado-allí.PROX 2 REL $=3 \mathrm{COP}=$ salir PREP.LOC $=$ Pastores luego=3FUT=bajar $\quad$ DIR=abajo 'Aquí donde salían a Pastores, luego bajaban por abajo.'


En esta sección se ha descrito que el verbo intransitivo de mà?a exhibe el sentido de movimiento direccional; aparece con un complemento en forma de adjunto que denota una locación; igualmente, este verbo aparece con formas léxicas en donde codifica dirección. Las formas léxicas con à y mà a pueden aparecer, en diferentes contextos oracionales, con la misma palabra léxica com complemento. Se establece una relación de locación-tema; es decir, à y mà?a expresan la locación y la palabra léxica el tema.

\section{Formas léxicas}

En la lengua se encuentran formas léxicas formadas por la preposición, el verbo de movimiento que codifica marca de dirección y un enclítico de distancia, como àmàba de (30a), àmăk $k^{w} a$ de (30b) y àmànu de (30c). Es estas formas à codifica locación, mà dirección y el enclítico el grado de distancia. Por su parte en, màmànu de (30d), la marca de dirección se duplica. Recuérdese que las formas *mabə, *mak ${ }^{w} a$ y *manu no se realizan de forma autónoma, se les debe adjuntar $\grave{a}$ o mà.

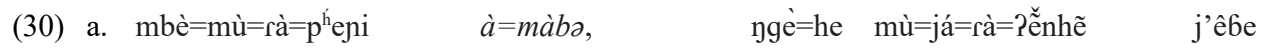
pero $=$ cuando $=3 \mathrm{FUT}=$ brillar $\mathrm{LOC}=$ DIR:aquí.PROX $1 \mathrm{ser}=\mathrm{LOC} \quad$ cuando $=\mathrm{PTL}=3 \mathrm{FUT}=$ venir lluvia 'Pero cuando refleja por acá, es cuando va venir lluvia.'

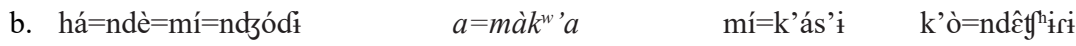
donde $=$ mitad $=3 \mathrm{COP}=$ caminar $\quad$ LOC $=$ DIR:allí. $P R O x 23 \mathrm{COP}=$ cuidar $\quad$ DEMPL $=$ borrego 'Dondequiera andaba por allí, cuidaba esos borregos.'

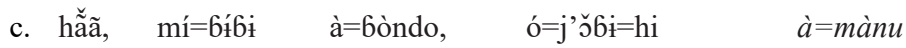
$\mathrm{AF} \quad 3 \mathrm{COP}=$ vivir $\quad$ LOC=México $\quad 3 \mathrm{PST}=$ enterrar $=\mathrm{PL} \quad$ LOC $=$ DIR:allá.PROX3 'Sí, vivía en México, la enterraron por allá.'

d. $m b o=\varnothing=\mathrm{k}^{\mathrm{h}} \hat{\mathrm{o}} 6 \mathrm{i} \quad \mathrm{i}=\mathrm{j}$ 'óbe, $\quad \varnothing=\mathrm{k}^{\mathrm{h}} \hat{\mathrm{o}} 6 \mathrm{i} \quad$ mà=mànto, $\quad$ mà=mànu luego=3PST=pasar $\quad 1 \mathrm{POS}=$ hermano $3 \mathrm{PST}=$ pasar $\quad$ ir.MOV=Manto $\quad$ DIR=DIR:allá.PROX3 'Luego pasó mi hermano, pasó por Manto, por allá

La forma $a$ igualmente aparece en formas léxicas que denotan modo, como $\grave{a} k^{h} \grave{a} n u$ de (31a), y $\grave{a} k^{h} \grave{a} k^{w}$ 'a de (31b); estas están formadas por la marca de locación $a$, el morfema $k^{h} a$ que expresa modo y un enclítico de distancia. En algunos contextos estas mismas formas, como $\grave{a}=k^{h} \grave{a} b$ o en (31c), también codifican locación. En estos casos los dos morfemas, $k^{\prime} a$ y $k^{h} a$, aparecen para expresar locación.

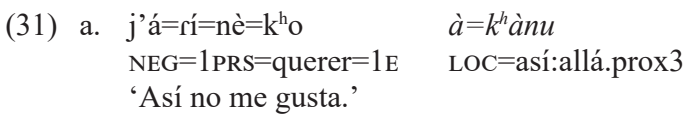

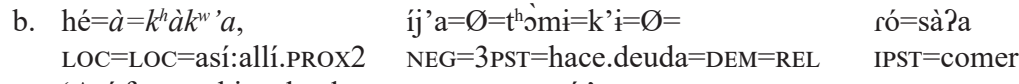
'Así fue, no hice deuda con eso que comí.' 


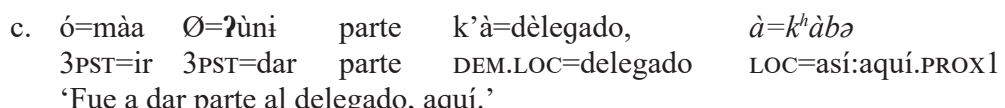

En los ejemplos de (32a) y (32b), la forma léxica $k^{h} \grave{a} b$ a aparece marcada con el artículo de énfasis o relieve nù; esta marca implica que el sentido denotado por la forma léxica aparezca en primer plano, ya que está altamente identificado en el acto enunciativo. Este artículo de énfasis, relieve o foco es muy productivo en otras variantes del mazahua (Steward 1966); en la variante que aquí se describe $\mathrm{y}$ en el corpus que se dispone, esta forma sólo aparece en contextos como los que se ilustran en (32).
a. ná= $\mathrm{p}^{\mathrm{h}} \mathrm{a} d \mathrm{~d}=\mathrm{k}$ 'i
¿há=rì=tshă-pí=k'ì?
rá=mà $\quad \varnothing=\mathrm{k}^{\text {hăa }} \quad$ ndéhe
PRED.PRS $=$ caballo $=$ DEM
como $=2$ FUT $=$ hacer -3 DAT $=$ DEM
$1 \mathrm{FUT}=$
¡Ø $=\int$ óki $^{\mathrm{h}} \mathrm{t}^{\mathrm{h}} \mathrm{O}$
$n \grave{u}=k^{h} a b ə$ !
3.FUT $=$ IMP.abrir $=$ DEL
ART.E=así:aquí.PROX 1
'Es canijo ese ¿Qué le vas a hacer a ese? Voy al baño. ¡Abre aquí nomás!'

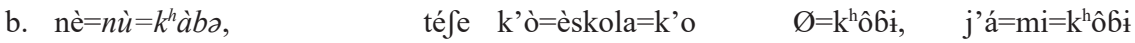 $\mathrm{CONJ}=\mathrm{ART} . \mathrm{E}=$ así aquí.PROX 1 todo $\mathrm{ART} . \mathrm{PL}=$ escuela $=\mathrm{REL} \quad 3 \mathrm{COP}=$ pasar $\quad \mathrm{NEG}=3 \mathrm{COP}=$ pasar 'Y aquí, a todas las escuelas que pasaba, no aprobaba.'

Dentro del repertorio de formas que expresan locación, el mazahua dispone de un conjunto de unidades léxicas que están formadas por los proclíticos $a ̀$ o

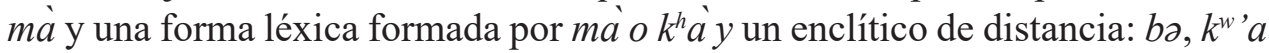
o $n u$. También aparece un morfema que expresa realce sobre la locación.

\section{Locativos y coocurrencias}

Dentro del repertorio de la frase prepositiva locativa, la lengua presenta construcciones, como en (33), en donde el complemento es un verbo con su respectivo adjunto. Las frases prepositivas locativas àmánéndzare 'por la orilla del rio' de (33a) y àmàbòndo 'por México' de (33b), están formadas por el núcleo prepositivo à y el complemento oracional mànèndzare y màbòndo. Este complemento, como fue señalado más arriba, es una oración integrada por el verbo de movimiento con dirección mà'a 'ir' y un adjunto: nèndzare 'orilla del rio' y 6òndo 'México' que funge como complemento. Dentro del repertorio de adjuntos del verbo mà?a aparece una frase nominal locativa, como en (33c), $k$ 'a ndzùmi 'en la casa'. Parecería que esta clase de adposición es rara en su configuración, sin embargo, Hagège (2010) considera que puede aparecer en otras lenguas del mundo.

(33) a. rà=mò $=\mathrm{hi} \quad \grave{a}=m a \grave{a}=n e ́ n d b-a r e$

2FUT $=$ ir $=$ PL $\quad$ PREP.LOC $=$ ir.DIR $=$ orilla-río

'Vayan por la orilla del río.' lit: vayan allá, por la orilla del río 
b. rá=mà?a $\quad \grave{a}=m \grave{a}=6 \grave{o} n d o, \quad$ rá=mà $\quad \emptyset=\int \grave{r} \dot{r}$ 1FUT $=$ ir $\quad$ PREP.LOC $=$ ir.DIR $=$ México $\quad 1 \mathrm{FUT}=$ ir $\quad$ 1FUT $=$ estudiar

'Voy a ir por México, voy a estudiar.' Lit: voy a ir allá, por México, voy a estudiar
c. j'à= $=\mathrm{k}^{\mathrm{h}} \mathrm{o} \quad \varnothing=$ nè?e $\quad$ rò=mà?a $\quad \varnothing=$ ndłódł NEG $=$ SUB $2 \mathrm{PST}=$ querer $3 \mathrm{PST}=\mathrm{ir} \quad 3 \mathrm{PST}=$ caminar

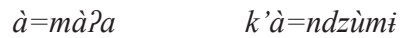 'No quiero que nadie vaya a caminar por la casa.'

En (34) coocurren una frase prepositiva locativa y una frase nominal igualmente locativa; la relación que se establece entre entidades es del tipo locación-locación (tema). Las dos entidades expresadas en estos recursos sintácticos están yuxtapuestas, no aparece marca gramatical alguna que exprese el tipo de relación que se presenta; ninguna frase está por encima de la otra, simplemente la frase prepositiva que tiende a ubicarse antes de la frase nominal exhibe la locación y la frase nominal, con el demostrativo locativo, expresa el tema.

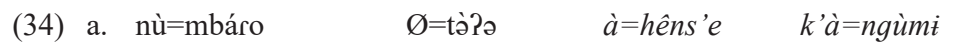 ART.RCB $=$ paloma $\quad 3$ PRS $=$ parar $\quad$ LOC $=$ arriba $\quad$ DEM. $. \mathrm{LOC}=$ casa 'El pato está parado arriba del árbol.'

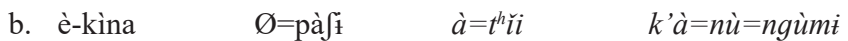 MV-Macrina 3PRS $=$ barrer $\quad$ LOC $=$ afuera $\quad$ DEM.. LOC $=A R T . R C B=$ casa
'Macrina barre afuera de la casa.'

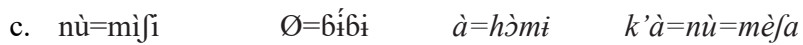 ART.RCB $=$ gato 3 PRS $=$ estar $\quad$ LOC $=$ tierra $\quad$ DEM.LOC $=$ ART $=$ mesa 'El gato está debajo de la mesa.'
d. tá $=\varnothing=6 \hat{t}^{\prime} \dot{\mathrm{i}}=\mathrm{nu} \quad k^{\prime} \grave{a}=\int o^{\prime} n i \quad k^{\prime} \grave{a}=t a ̀ p a n k o$ hasta $=3$ PRS $=$ estar=allá.PROX 3 DEM.LOC $=$ arriba $\quad$ DEM.LOC $=$ tapanco 'Está hasta allá, arriba en el tapanco.'

En una construcción es natural encontrar más de una frase prepositiva o de una frase nominal locativa; también, en la misma oración estas pueden coocurrir junto con el verbo que expresa movimiento direccional. En (35a) coaparecen dos frases prepositivas locativas àkàrakole 'en Caracoles' àfó 'ni 'arriba' y una forma léxica àmànu 'por allá'. La frase prepositiva, àfó 'ni 'arriba', precisa la locación, mientras que la forma léxica àfó'ni exhibe la dirección. En (35b), coaparecen las frases nominales locativas, k'à èskola, k'àfàbrika y la frase prepositiva locativa àmbàro. Los dos últimos adjuntos subespecifican al primero que semánticamente se le asigna el papel temático de meta. En (35c), la forma sintáctica que expresa el adjunto es una oración, mà $k^{\prime} \grave{a} \int \hat{t}^{h} h^{h} k^{\prime}$ à $n d z u m \dot{t}$, el complemento del predicativo verbo mà?a, es una fase nominal locativa compleja, $k^{\prime} \grave{a} \int_{\hat{t}} h^{h} \dot{t} k^{\prime} a ̀ ~ n d z u m i t$, la cual está formada por un núcleo nominal $n d z u m i$ modificado por un demostrativo locativo $k$ 'à, un locativo léxico $\hat{t}^{h} t^{h} y$ el demostrativo locativo de primera posición de frase $k$ 'à. 


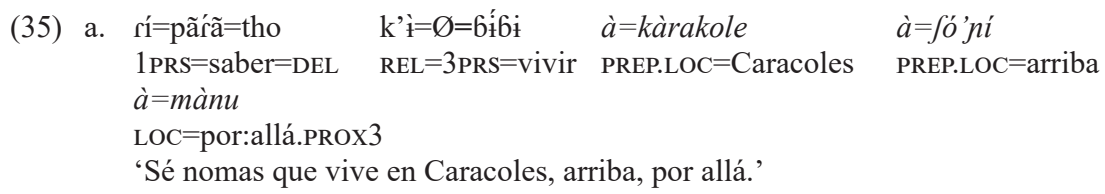

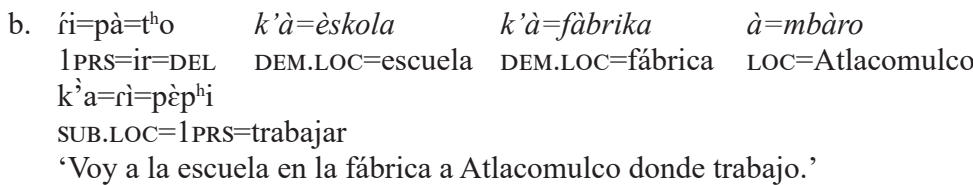
c. $m \grave{a}=k^{\prime} \grave{a}=\int \hat{t} t^{h \grave{i}} \quad k^{\prime} \grave{a}=n d z \grave{u} m \dot{t} \quad$ ò $=$ rèklama; $\quad$ ò=?е̃̃̃ $\quad$ mí=hjoo ir.DIR $=$ DEM.LOC $=$ atrás $\quad$ DEM.LOC $=$ casa $\quad 3 \mathrm{PST}=$ reclamar $\quad 3 \mathrm{PST}=$ venir $\quad 1 \mathrm{POS}=$ papá 'Por atrás de la casa reclamó; vino mi papá.'

En el sistema de formas locativas no verbales, dentro de una oración pueden coaparecer varias formas locativas de la misma o diferente naturaleza gramatical. Algunas de estas formas coocurren para hacer énfasis sobre el referente de la locación, se yuxtaponen para explicitar la relación que se establece entre estas entidades locativas, o simplemente se trata de frases nominales locativas o frases prepositivas complejas que presentan una estructura gramatical específica.

\section{Conclusiones}

En este trabajo se han descrito los diferentes recursos gramaticales no verbales que dispone la lengua para expresar la locación; se ha presentado un panorama de los morfemas libres y ligados que codifican diferentes grados de distancia con respecto al punto deíctico. Los morfemas ligados se adjuntan en el verbo o en alguno de los argumentos o adjuntos, los morfemas libres o léxicos se ubican al principio o al final de la oración; en los dos casos el alcance o dominio es sobre el núcleo verbal o alguno de los constituyentes de la oración.

Con respecto a nivel frasal y oracional son tres los mecanismos sintácticos que expresan locación; la frase nominal locativa está formada por un núcleo nominal y una serie de operadores que lo modifican; estructuralmente en estas frases están implicadas las marcas de referencia y de locación. Estas frases están tipificadas básicamente por un demostrativo locativo que se ubica en primera posición de frase. La frase prepositiva está integrada por el núcleo prepositivo y un complemento nominal, cuya referencia debe ser conocida o compartida por los interlocutores del acto de habla, igualmente, la única preposición de la lengua que codifica locación aparece con un complemento verbal; el tercer recurso que dispone la lengua para expresar locación es un verbo que expresa un sentido de dirección; este predicado aparece con un complemento que funciona como su adjunto.

Un conjunto de unidades léxicas coocurren tanto con la preposición como con el verbo de dirección y generan formas lexicalizadas y expresan locación; 
en estas formas la preposición y el verbo no se constituyen como unidades nucleares, simplemente se exhiben como marcas de locación y dirección respectivamente.

En estudios posteriores se debe tener en consideración la naturaleza sintáctica, semántica y pragmática de todos los recursos no verbales de la lengua para codificar locación. Esto en el sentido estricto de explicar las coocurrencias que se presentan entre las unidades morfológicas, léxicas, frasales y oracionales. Sin ir muy lejos salta a la vista la inquietante pregunta sobre la relación de los conceptos, el fondo y la figura, ya clásicos sobre el tema de la locación. Téngase en cuenta que con este trabajo se hace una sugerencia, en torno a la locación no verbal, de los recursos gramaticales que dispone la lengua.

\section{Abreviaturas}

Abreviaturas $: \mathrm{AF}=$ afirmación; $\mathrm{AMB}=$ ambulativo; $\mathrm{ART}=$ artículo; $\mathrm{ASP}=$ aspecto CIS $=$ cislocativo $; \mathrm{CONJ}=$ conjunción $; \mathrm{COP}=$ copretérito; $\mathrm{DAT}=$ dativo; $\mathrm{DEF}=$ definido; $\mathrm{DEL}=$ delimitativo; $\mathrm{DEM}=$ demostrativo; $\mathrm{DIM}=$ diminutivo; DIR $=$ direccional $; \mathrm{DU}=$ dual $; \mathrm{E}=$ enfático $; \mathrm{EST}=$ estativo $; \mathrm{EXCL}=$ exclusivo $; \mathrm{FUT}=$ futuro; $\mathrm{IDF}=$ identificativo; $\mathrm{IMP}=$ impersonal; $\mathrm{LEX}=$ léxico; $\mathrm{NEG}=$ negación; NOM $=$ nominal $;$ MOV $=$ movimiento $;$ MV $=$ marca de valoración $; \mathrm{o}=$ objeto; $\mathrm{PL}=$ plural $; \mathrm{POS}=$ posesivo $;$ PRED $=$ predicativo $;$ PREP $=$ preposición $;$ PRON $=$ pronombre; PROX $=$ proximidad; $\mathrm{PRS}=$ presente; $\mathrm{PST}=$ pasado; $\mathrm{PTL}=$ puntual; $\mathrm{QU}=$ pregunta $; \mathrm{RCB}=$ reconocible $; \mathrm{REC}=$ receptivo; $\mathrm{REL}=$ relativo; $\mathrm{SUB}=$ subordinanate; $\mathrm{TADV}=$ tiempo adverbial; $\mathrm{TPR}=$ tópico de referencia.

\section{Referencias}

Ameka, Felix, y Levinson Stephen. 2007. Introduction- The typology and semantics of locative predicates: posturals, positionals and other beasts. Linguistics 45, 847-871.

Bohnemeyer, Jurgen y Penelope Brown. 2007. Standing divided: dispositionals and locative predication in two Mayan languages. Linguistics 45, 1105-1151.

Dryer, Matheus y Martín Haspelmath. (eds.). 2013. The World Atlas of Language Structures Online. Leipzig: Max Planck Institute for Evolutionary Anthropology.

García Zúñiga, Antonio; Nathalia Hernández Hernández; Alejandra Ortiz Villegas y Armando Mora-Bustos. 2019. Adposiciones y sistemas mixtos en tres lenguas otomangues. Comunicación presentada en el Seminario de diacronía y adposiciones: origen y evolución'. Universidad de Sonora. Hermosillo. 14-15 de noviembre.

Guerrero, Lilian (ed.). 2019. Adposiciones y elementos de su tipo en lenguas de América. México: Universidad Nacional Autónoma de México.

Guillaume, Antoine. 2017. Sistemas complejos de movimiento asociado en las lenguas Takana y Pano: perspectivas descriptiva, tipológica e histórico-comparativa. Amerindia 39, 211-261. 
Hagège, Claude. 2010. Adpositions. Oxford: Oxford University Press.

Hemphill Chisty y Aoron Hemphill. 2019. Funciones adposicionales y palabras múltiples categorías en me'phaa. En Lilian Guerrero (ed.). Adposiciones y elementos de su tipo en lenguas de América. México: Universidad Nacional Autónoma de México. 277-314.

Hernández Chincoya, Francisco y Armando Mora-Bustos. 2019. Adverbios por posposiciones en chichimeco. Comunicación presentada en el Seminario de diacronía y adposiciones: origen y evolución'. Universidad de Sonora. Hermosillo. 14-15 de noviembre.

Knapp, Michael. 2008. Fonología segmental y léxica del mazahua. México: Instituto Nacional de Antropología e Historia.

Levinson, Stephen. 2003. Space in Language and Cognition. Explorations in Cognitive Diversity. Cambridge: Cambridge University Press.

Levinson, Stephen, y David P. Wilkins. 2006. The background to the study of the lenguage of space. En Stephen Levinson y David P. Wilkins (eds.). Grammars of space, Cambridge: Cambridge University Press, 1-23.

Lillehaugen, Brook Danielle.99 2006. Expressing location in Tlacolula Valley Zapotec. Tesis de doctorado. University of California. Los Angeles.

Lizárraga Navarro, Glenda y Armando Mora-Bustos. 2015. Gramática de los adjuntos. Estudios de Lingüística Aplicada, 33, 225-251.

Mora-Bustos, Armando y Mora Muñoz, Gabriela. 2018. La estructura de la frase nominal en mazahua. Estudios de lingüística aplicada. 36, 131-174.

Stewart, Donald. 1966. Gramática del mazahua. Correcciones y comentarios de Doris Bartholomew (Manuscrito). México: Instituto Lingüístico de Verano.

Van Valin, Robert, Jr y Randy LaPolla. 1997. Syntax: structure, meaning and function. Cambridge: Cambridge University Press. 